\title{
LATTICE MEASUREMENT FOR FERMILAB MAIN INJECTOR
}

\author{
M.J. Yang ${ }^{\ddagger}$, Fermilab $^{\dagger}$, Batavia, IL 60510, USA
}

\begin{abstract}
The installation of seven large aperture quadrupoles during shut-down of 2006 necessitates new lattice measurements to evaluate the state of the machine lattice at energies critical to operation. For Main Injector (MI) this means at $8-\mathrm{GeV}$ kinetic energy for proton and pbar injections, at $150-\mathrm{GeV}$ extraction to Tevatron for collider operation, and at $120-\mathrm{GeV}$ extraction to Numi for nutrino experiment and slow spill to fixed target test beam facility. The results of measurement at these three energies and their comparison to their corresponding calculations will be presented.

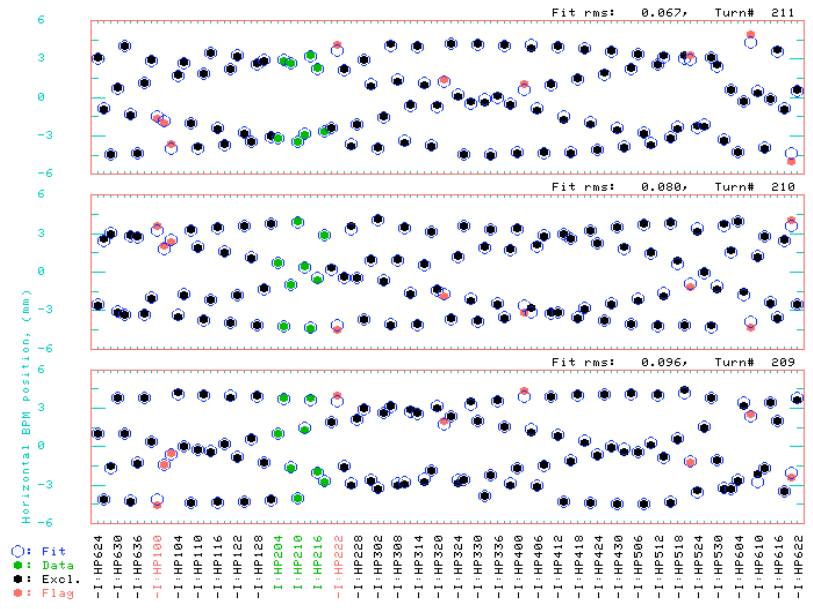

Figure 1. Example of horizontal plane TBT data, ring-wide and for 3 consecutive turns, from $8-\mathrm{GeV}$ measurement.

\section{INTRODUCTION}

The Fermilab Main Injector ring was originally built with three different types of quadrupole magnets, i.e. IQB of 84 inches, IQC of 100 inches, and IQD of 116 inches. New type B wide aperture quadrupoles (WQB) were installed during shut-down of 2006 to replace existing IQB magnets at seven injection or extraction locations. Magnetic measurement indicated that they are appreciably different from IQB magnets. With all quadrupoles powered by either focusing or de-focusing bus the use of built-in trim coils for correction was considered necessary. Lattice functions measurement would be used to evaluate overall impact of WQB magnet compensations and to ensure that there were no surprises.

In addition to installing wide aperture quadrupoles the Main Injector BPM read-back system was also upgraded and was commissioned in the few months after beam operation returned. The new system is more reliable and provides much better position resolution. It has a built-in 2048 turns TBT data buffer. Figure 1 shows an example

\footnotetext{
$\uparrow$ Operated by Fermi Research Alliance, LLC under Contract No. DEAC02-07CH11359 with the United States Department of Energy.

$\ddagger$ E-mail: yang@fnal.gov
}

of data from horizontal plane $8-\mathrm{GeV}$ measurement.

Separate lattice measurements were carried out at 8 , 120 , and $150 \mathrm{GeV}$ of kinetic energies. Analysis results were compared with that of model calculations assuming perfect compensation for the new WQB quadrupoles.

\section{DATA}

There were originally 104 horizontal plane and 104 vertical plane BPMs, at corresponding focusing and defocusing locations. The installation of seven wide aperture dual-plane BPMs, at each wide aperture quadrupole, increased the number to 106 and 109, i.e. two horizontal at defocusing locations and five vertical at focusing locations. Extraction kicker at MI52 was used to excite free betatron oscillation in horizontal plane. The proton injection kicker at MI10 was used to excite vertical oscillation, with limited strength at 120 and $150 \mathrm{GeV}$ energy.

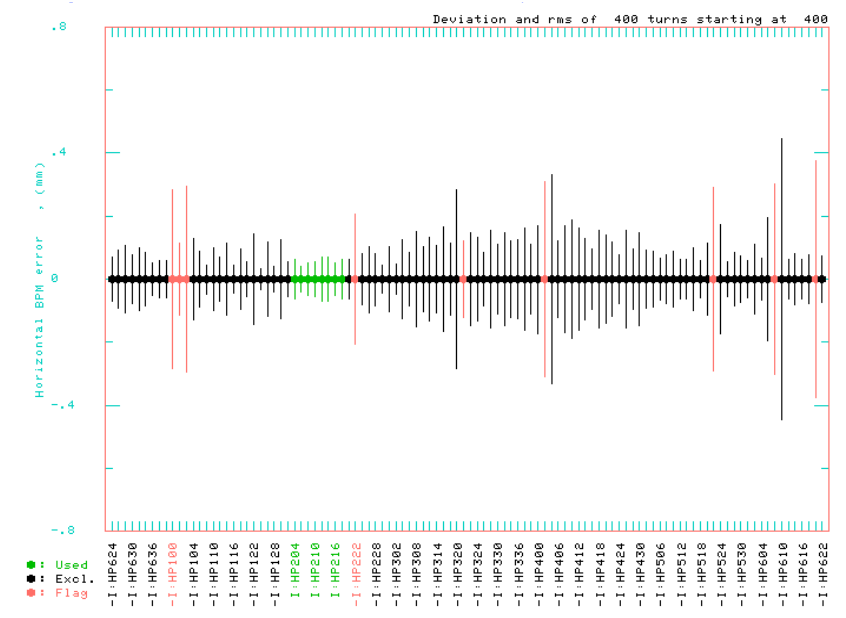

Figure 2. RMS errors from $8-\mathrm{GeV}$ horizontal TBT data. For each BPM the deviation between its reading and projected position at each turn is sampled and RMS calculated over 400 turns.

\section{ANALYSIS}

TBT lattice analysis [1] has been reported before. There are two basic steps in the process. The first is to fit for position and angle at the BPM of interest using data from its neighboring BPMs, and produce one phase space point for every turn. Phase space data points from a number of consecutive turns are then fitted to an ellipse, whose parameters are used to calculate lattice functions, $\beta$ and $\alpha$.

\section{Fitting for Phase space coordinates}

Shown in Figure 1 are ring-wide horizontal plane TBT data for three consecutive turns. Horizontal position at HP210 is the target of interest. In this example data from eight BPMs were used in fitting and plotted as green dots. Other BPM data are plotted as black dots to show contrast with projected positions. The fit produces a pair of $x$ and $x$ ' horizontal phase space coordinates for every turn.

The projected positions for all BPMs are also shown as 
circles in Figure 1. Given fitted result projections are calculated based on design lattice. While good agreements between green dots and circles are expected they are not at all guaranteed with other data points, if design lattice and reality are substantially different. At each BPM such deviations were sampled, over some number of turns, and their RMS calculated. Plotting such RMS for all BPMs, as shown in Figure 2, is a quick way to find out if gross errors in either data or analysis setup is present.

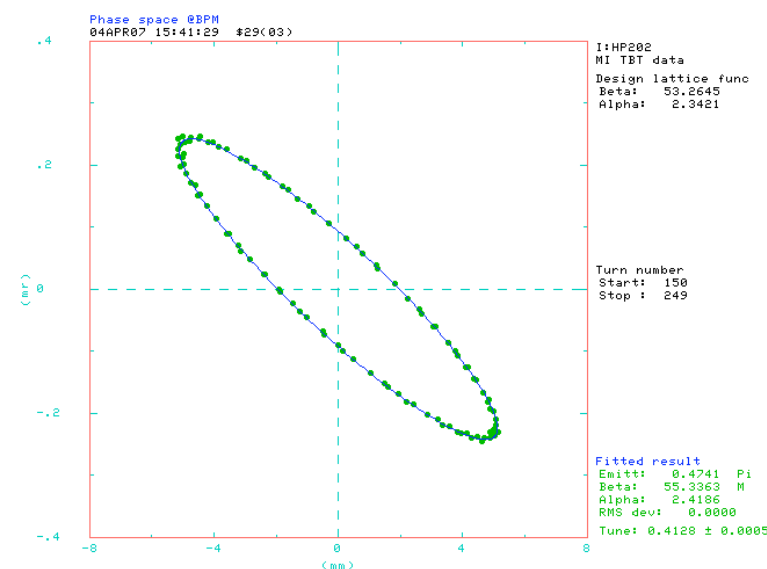

Figure 3. Plot of horizontal phase space points at HP202 for 100 consecutive turns, from $120-\mathrm{GeV}$ TBT data. The measured lattice functions are shown at lower-right corner and the design lattice functions at the upper-right corner.

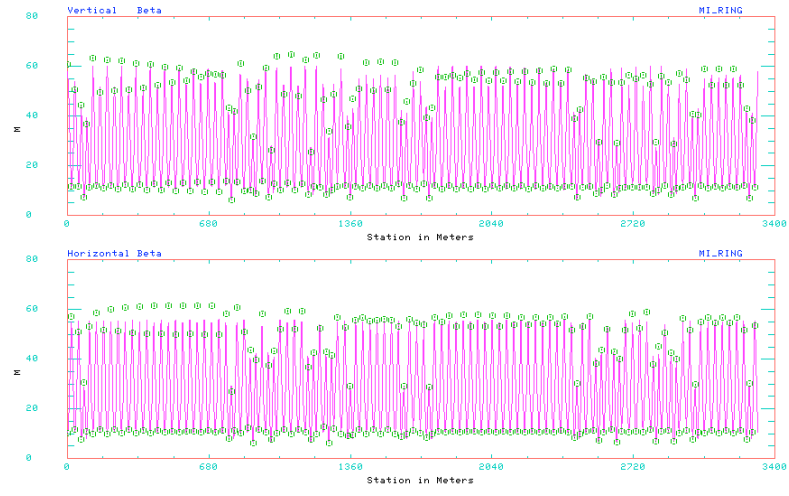

Figure 4. Measured Main Injector $8-\mathrm{GeV}$ beta function. Bottom plot is for horizontal plane and top plot vertical plane. Green circles are measured beta functions and magenta traces are the design values. Statistical beta errors are plotted as vertical lines, i.e. the barely visible short vertical lines inside the circles.

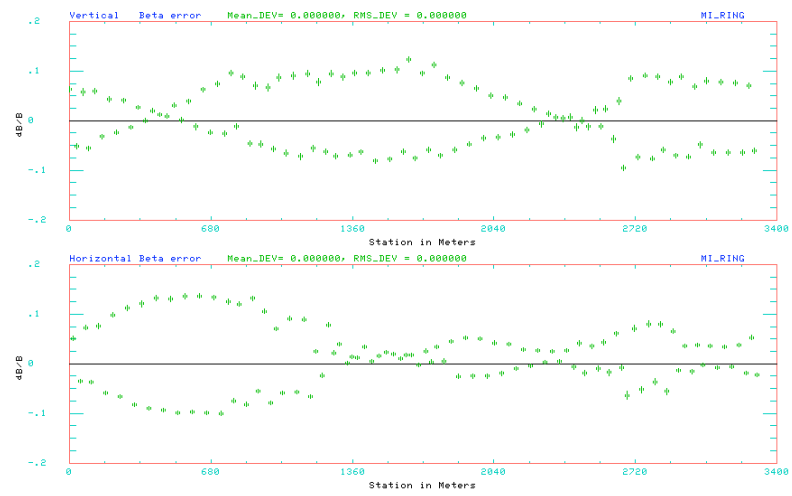

Figure 5. Beta error of measured MI $8-\mathrm{GeV}$ beta functions. Plotted are $\Delta \beta / \beta$, i.e. deviation divided by design value.

\section{Fitting ellipse}

Figure 3 shows phase space points at HP202 for 100 consecutive turns. The elliptical path traced out by these data points are dictated by actual beta and alpha functions. The fitted ellipse is shown in blue trace. Its parameters are used to calculate measured beta and alpha functions, as listed at the lower-right corner. The design lattice functions are also listed at the upper-right corner. The area encompassed by the ellipse, listed as emittance, is obtained as part of the fitting algorithm.

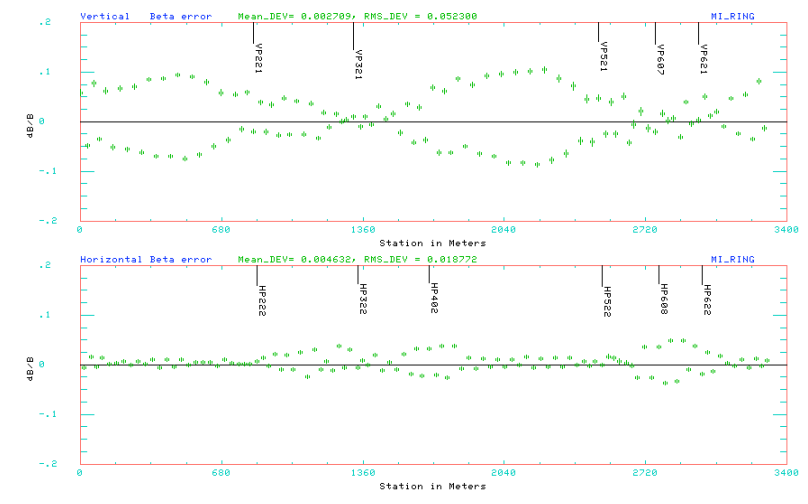

Figure 6. Beta error for MI $120-\mathrm{GeV}$ beta function. While maximum horizontal error is down to $5 \%$, maximum vertical errors are still at $10 \%$.

\section{LATTICE RESULTS}

\section{At 8-GeV injection porch}

The measured Main Injector $8-\mathrm{GeV}$ beta functions are plotted in Figure 4, the horizontal plane at the bottom and the vertical plane on top. The design beta functions are also shown in magenta trace. Some differences between measurement and design are clearly visible. The deviations can be seen more directly in Figure 5 where fractional beta error $\Delta \beta / \beta$ are plotted. Maximum beta error is about $15 \%$ in horizontal plane and $10 \%$ in vertical plane.

\section{At 120-GeV flat-top}

The lattice measurement result at $120 \mathrm{GeV}$ is shown in Figure 6 , only for the deviations in $\Delta \beta / \beta$. The agreement between measurement and design in the horizontal plane is excellent at 5\% level, while the agreements in the vertical plane remains about the same at $10 \%$ level.

\section{At 150-GeV flat-top}

This is the highest energy Main Injector was designed to operate at and, in some way, the machine is acting a bit different. The result of lattice analysis revealed that the beta error in the horizontal plane is substantially larger. Figure 7 shows the same beta error plot as before. However, the scale for the horizontal error, the bottom plot, has been expanded from \pm 0.2 to \pm 0.4 . Maximum beta error in the horizontal plane is now $25 \%$, while the vertical plane is still at $10 \%$ level. A hint of horizontal problem can be seen in Figure 8. The bowtie shaped RMS plot, to be compared with Figure 2 of $8-\mathrm{GeV}$ measurement, says that the projected positions using design lattice 
are not very compatible with the real machine. The same conclusion can be made by looking at Figure 9, which shows that calculations are not matching data very well. Also shown in Figure 9 is the rms from fitting green data points for phase space coordinates. This number, shown at the top of each plot box, has gotten larger as compared with that from $8-\mathrm{GeV}$ data shown in Figure 1.

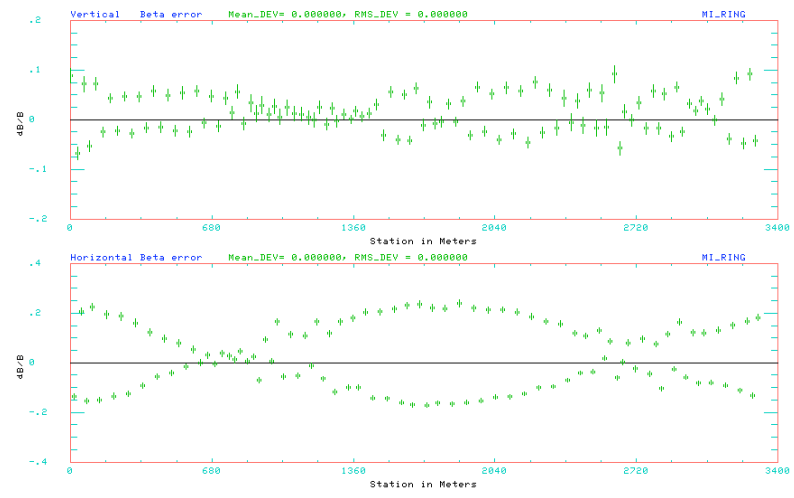

Figure 7. Beta error at $150 \mathrm{GeV}$. Maximum vertical error is below $10 \%$ and maximum horizontal error is over $25 \%$, bottom plot with its vertical scale range doubled, to \pm 0.4 from \pm 0.2 .

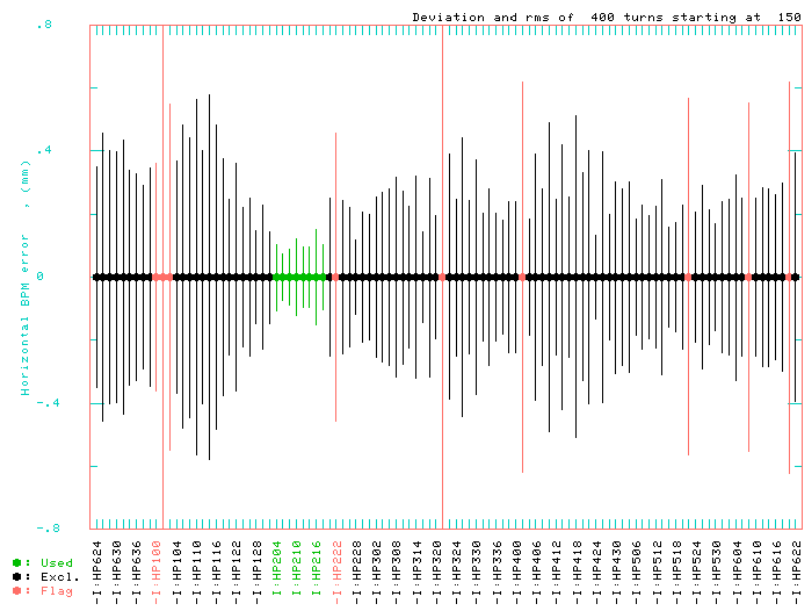

Figure 8. Horizontal plane RMS error plot from MI $150 \mathrm{GeV}$ measurement. Small RMS for data points in green is expected because they influence fitting process. Outside green zone the projected positions no longer match data and RMS jumped.

\section{WQB MAGNETS AND BETA ERRORS}

As mentioned at the beginning, part of the motivation to conduct the studies was to understand if systematic error from WQB magnets is present. This is not to say that there isn't any beta function errors already in the machine.

Remnant field due to hysteresis is the most likely error at $8-\mathrm{GeV}$ energy. This is typically addressed by ramping magnet current sufficiently below operating current and come up to $8-\mathrm{GeV}$ injection porch at an up-ramp. WQB magnets were measured and its hysteresis behavior compensated with its trim coils to make it behave exactly like an IQB magnet. The beta wave pattern observed is not consistent with systematic error in WQBs.

At $150 \mathrm{GeV}$, on the other hand, magnet saturation would be the primary issue. As has been demonstrated, the horizontal plane beta wave is quite real. Lattice measurement from 2004, when there were no WQB magnets installed, also showed telltale sign of what is now clear picture of beta error. At that time data quality was much worse and was hard to take action on. With all likelihood the lattice problem is not associated with WQB magnets.

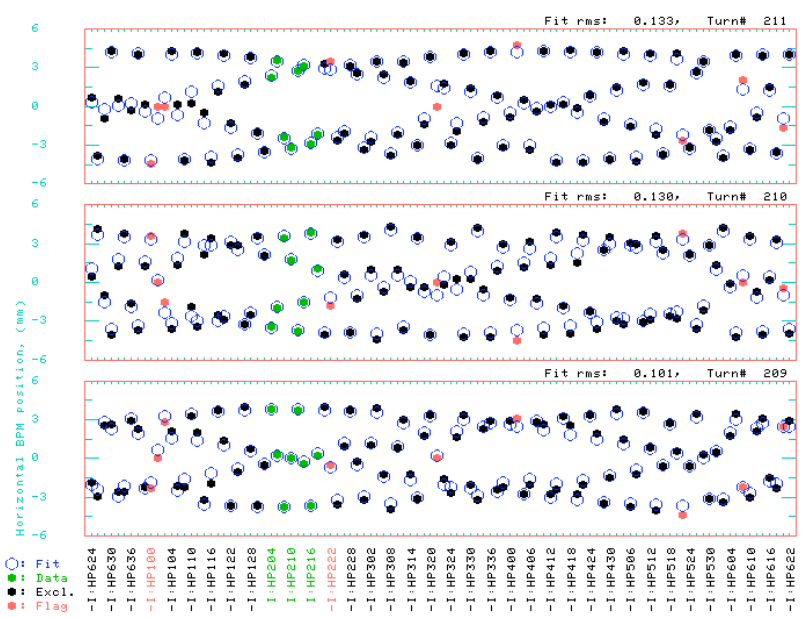

Figure 9. Horizontal plane TBT data from $150 \mathrm{GeV}$ measurement. Agreements between projections and data are visibly poor. This is a complimentary view of what was shown in Figure 8.

\section{CONCLUSION}

The study results presented here indicate errors in Main Injector lattice function. However, evidence does not point to systematic error from the newly installed WQB magnets. The worst error is found in the horizontal plane at $150 \mathrm{GeV}$ energy. The cause of this is still being investigated. The Main Injector dipole does saturate substantially at this energy. Magnet data is been reviewed to provide further insight. A linear optics model has been used for calculations throughout and may well be inadequate.

TBT lattice analysis has been shown to produce results that are reflective of the machine lattice over the years. Only recently the measured beta functions are used in TBT BPM gain calibration [2] with great success. This gives even more credence to the analysis technique. It is still possible that analysis is at fault at some level. More effort and studies will be needed to get to cause of error.

\section{ACKNOWLEDGEMENT}

The author would like to thank D.E. Johnson for many useful discussions, B.C. Brown and D. Harding for consultation on magnet measurement data, the Femilab Computing Division and the instrumentation department of Accelerator Division for an excellent new BPM system.

\section{REFERENCES}

[1] M.J. Yang, "Lattice Measurement with TBT BPM Data", PAC 1995, pp. 2500.

[2] M.J. Yang, J. Crisp, P. Prieto, “A BPM Calibration Procedure using TBT Data”, this proceeding. 